\title{
APLICABILIDADE DA LÓGICA NEBULOSA NO PROCESSO DE DESLIGNIFICAÇÃO DO CAPIM-ELEFANTE NO PRÉ-TRATAMENTO COM NAOH
}

\author{
APPLICABILITY OF THE CLOUDY LOGIC IN THE DESLIGNIFICATION PROCESS OF \\ ELEPHANT GRASS IN THE PRE-TREATMENT WITH NAOH ${ }^{\circ}$ \\ APLICABILIDAD DE LA LÓGICA NUBLADA EN EL PROCESO DE DESLIGNIFICACIÓN \\ DE HIERBA ELEFANTE EN EL PRETRATAMIENTO CON NAOH \&
}

Recebido em: 17/10/2021 - Aprovado em: 10/11/2021 - Publicado em: 22/11/2021

d

http://dx.doi.org/10.18011/bioeng2021v15n3p497-510

Emmanuel Zullo Godinho' (emmanuel.godinho@unesp.br)

Fernando de Lima Caneppele ${ }^{2}$ (caneppele@usp.br)

Hélio Vagner Gasparotto' (hélio.vagner@unesp.br)

\footnotetext{
1 Universidade Estadual Paulista. Botucatu, SP, Brasil

2 Universidade de São Paulo. Pirassununga, SP, Brasil.
}

\section{RESUMO}

O capim-elefante apresenta grande potencial para a produção de bioetanol com destaque em produções a campo e manuseio industrial. Sendo que o processo de pré-tratamento na biomassa é fundamental para obter altos rendimentos na produção de bioetanol. Por isso, a otimização é fundamental para reduções de custos e aumento na rentabilidade financeira. Em virtude disso, objetivou desenvolver um sistema baseado em regras difusas, onde otimizou o processo de pré-tratamento com $\mathrm{NaOH}$ no capim-elefante. $\mathrm{O}$ modelo fuzzy foi desenvolvido utilizando duas variáveis de entrada e uma de saída, sendo concentração, temperatura e lignina, respectivamente. A análise dos efeitos da concentração e temperatura foi realizada por superfícies tridimensionais e mapas de contorno da variável de saída. Verificou-se que as variáveis relacionadas à lignina são amplamente afetadas por uma baixa dosagem da concentração do $\mathrm{NaOH}$ e com uma temperatura média a baixa. Em geral, conclui-se que a região entre 0,4 a $0,6 \%$ de concentração de $\mathrm{NaOH}$, combinado com uma temperatura entre 80,5 a $85,0^{\circ} \mathrm{C}$, verificam-se os melhores resultados para a variável biométrica estudada, a lignina.

Palavras-chave: Lignina. Hidróxido de sódio. Lógica Fuzzy. 


\section{INTRODUÇÃO}

A produção de energias renováveis vem se destacando a cada dia nos mercados mundiais, principalmente a produção de biocombustíveis como o bioetanol (PUSPAWATI et al., 2015). A principal matéria-prima utilizada é a biomassa (CARVALHO-NETTO et al., 2014). Biomassa são materiais provindos de restos oriundos de industrias, animais e principalmente vegetais (GODINHO et al., 2019).

O capim-elefante tem se destacado no mercado nacional na produção de bioetanol em usinas na região Nordeste do Brasil, por possuir duas características importantes em relação a cana-de-açúcar, a maior produtividade por área de plantio e a colheita com 6 meses após o plantio (GODINHO et al., 2019).

Nas biomassas vegetais são encontrados materiais lignocelulósicos, que é constituído de celulose, hemicelulose e lignina, conforme Behera et al. (2014), a porcentagem de celulose está entre 33 a $51 \%$, de hemicelulose 19 a $34 \%$ e a lignina 10 a $20 \%$. Mesmo possuindo baixa concentração em uma biomassa, a lignina é tem um papel fundamental na produção de bioetanol, isto se deve porque sua estrutura é extremamente rigida e tem como função o "esqueleteamento" da célula vegetal (RUEDA et al., 2016).

Para que suas estruturas possam ser removidas e consequentemente deixar a celulose mais exposta para o fungo na fermentação, é importante o uso do pré-tratamento (DEL Río et al., 2015). O pré-tratamento tem como objetivo remover ou modificar a estrutura da lignina para facilitar o ataque enzimático, evitando a degradação da célula (CARDONA et al., 2014). Entretanto, um dos "gargalos" na produção de bioetanol é o prétratamento, por ser de alto custo na produção citado por Cardona et al. (2013), sendo assim, a otimização no processo é fundamental, por buscar reduzir os custos diretos e indiretos para obter melhores rentabilidades financeiras.

A modelagem matemática é um método que é utilizado para otimizar processos produtivos industriais, agrícolas, econômicos, etc., Manekar et al. (2011) cita que um método eficiente é a aplicabilidade da lógica fuzzy. Caneppele et al. (2021), também apresenta a lógica difusa ou nebulosa, como uma metodologia importante e que trabalha com algoritmos que transcrevem uma seção do raciocínio humano.

Bhutto et al. (2015) e Godoy et al. (2020) descrevem que este método é operacionalizado por um programa baseado em um sistema de regras fuzzy, onde se aplica a matemática a conceitos difusos desenvolvido por Lotfi Asker Zadeh em 1965 e tenta 
Aplicabilidade da lógica nebulosa no processo de deslignificação do capim-elefante...

abordar o raciocínio humano por meio de conjuntos, também descritos por variáveis linguísticas.

Diversas são as aplicações da lógica fuzzy, principalmente no agribusiness, desde a geração de energias elétricas para aviários até mapeamento de solo para a agricultura. Por isso, desenvolveu-se um sistema baseado em regras difusas, onde otimizou o processo de pré-tratamento com $\mathrm{NaOH}$ no capim-elefante buscando maior desestruturação da lignina.

\section{MATERIAIS E MÉTODOS}

O artigo foi baseado em dados reais de laboratorio, buscando as melhores condições de deslignificação da lignina na biomassa do capim-elefante de Godinho et al. (2019), onde foram retirados os dados experimentais para modelagem da lógica fuzzy.

Foi desenvolvido um sistema baseado em regras difusas, onde estabeleceu buscar a melhor temperatura e concentração de $\mathrm{NaOH}$ no processo de pré-tratamento. Criou-se um processador de entrada (fuzzificador), um conjunto de regras linguísticas, um método de inferência fuzzy e um processador de saída (defuzzificador). A Figura 1 ilustra o sistema proposto com base em regras fuzzy.

Este processador possui uma ou mais entradas, conhecida como input e uma ou mais saídas output, onde foram definidos, conforme apresentado na Figura 1.

Figura 1 - Sistema baseado em regras fuzzy para a otimização do processo de pré-tratamento com $\mathrm{NaOH}$ do capim-elefante.

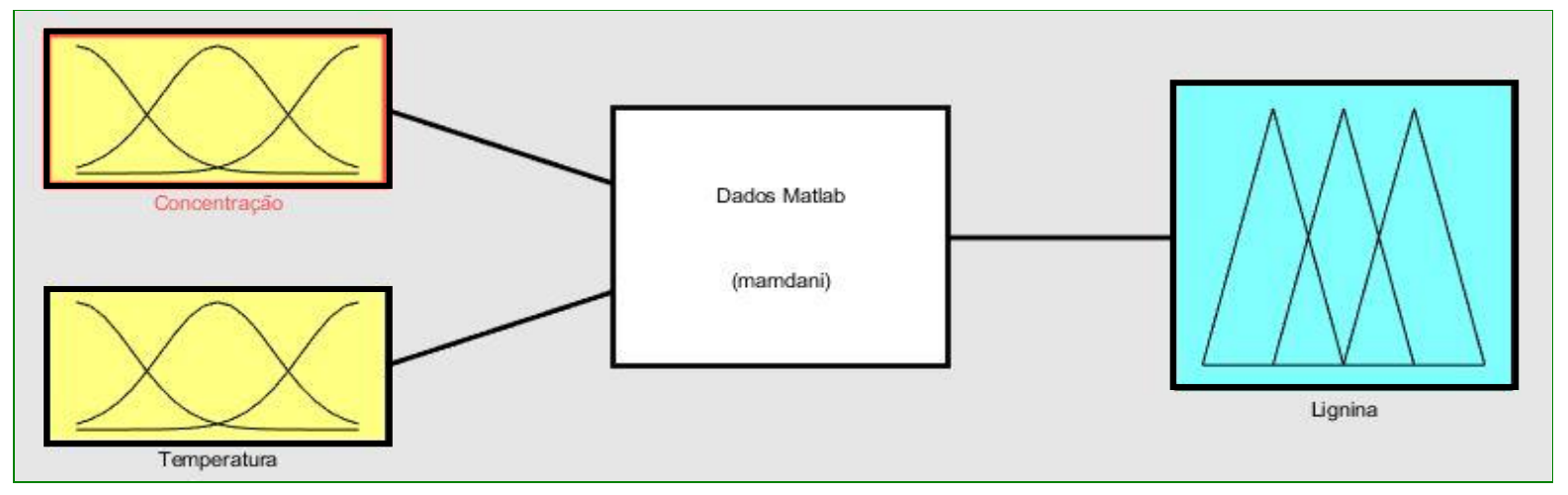

Fonte: Autor, 2021.

Existem diversas formas associadas as funções de pertinências que podem ser utilizadas para os dados de entrada, ou input e os dados de saída output. Conforme 
Godinho et al. (2019) a forma mais comum é a triangular, entretanto o modelo utilizado depende muito da preferência e experiência do projetista.

As funções de pertinência triangulares são caracterizadas por uma operacionalização simples utilizando siglas, podendo ser a, b e c. Sendo, o intervalo de a e c aplicados a valores diferentes de zero, e b é o ponto onde a função de pertinência é máxima.

Com isso, foi definido as principais funções de pertinência para a Temperatura e a Concentração de $\mathrm{NaOH}$ para o processo de deslignificação no processo de pré-tratamento do capim-elefante. Desenvolveu-se duas variáveis de entrada e, para cada entrada 3 graus de adesão, sendo baixa $(B)$, média $(M)$ e alta $(A)$. Os graus de pertinências para a Temperatura e Concentração é apresentado na Tabela 1 e nas Figuras 1 e 2.

Tabela 1 - Definições dos graus de pertinência para as variáveis Concentração e Temperatura.

\begin{tabular}{c|c|c|c}
\hline Conjunto difuso & Tipo & Concentração NaOH & Temperatura \\
\hline "Baixa" B & Triangular & {$[0,40,60,8]$} & {$[80,082,585,0]$} \\
\hline "Média" M & Triangular & {$[0,60,81,0]$} & {$[82,585,087,5]$} \\
\hline "Alta" A & Triangular & {$[0,81,01,2]$} & {$[85,087,290,0]$} \\
\hline
\end{tabular}

Fonte: Autor, 2021.

Figura 2 - Grau de associação para conjuntos difusos da variável da entrada - Concentração.

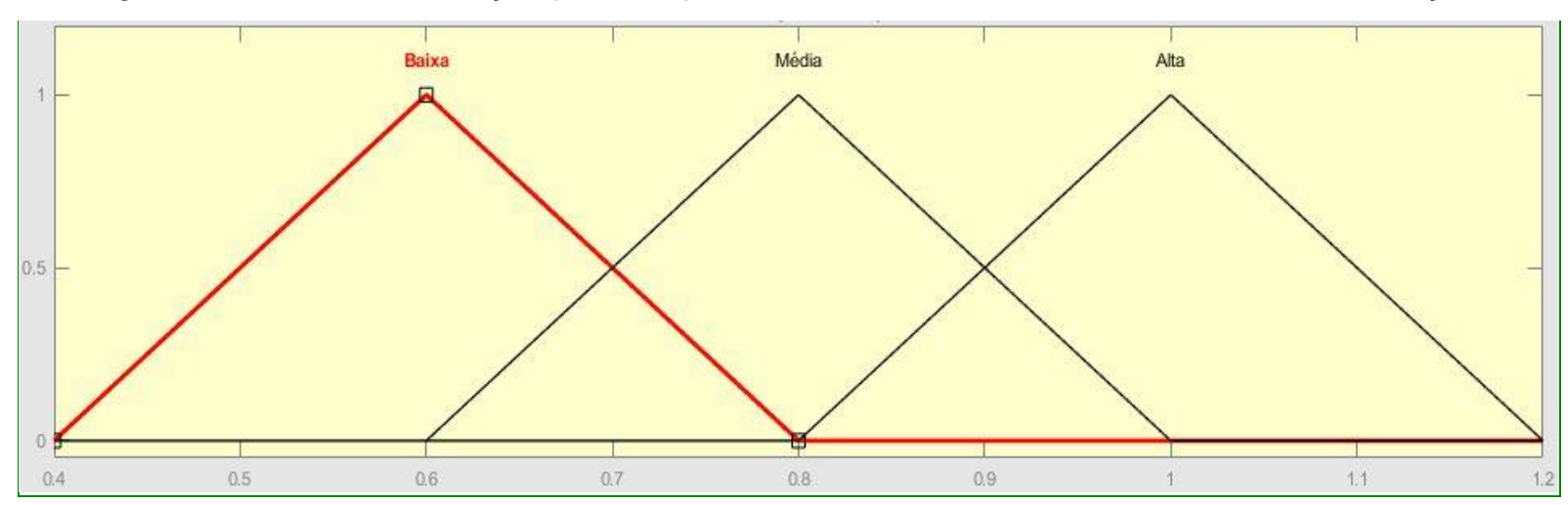

Fonte: Autor, 2021. 
Aplicabilidade da lógica nebulosa no processo de deslignificação do capim-elefante...

Figura 3 - Grau de associação para conjuntos difusos da variável de entrada - Temperatura.

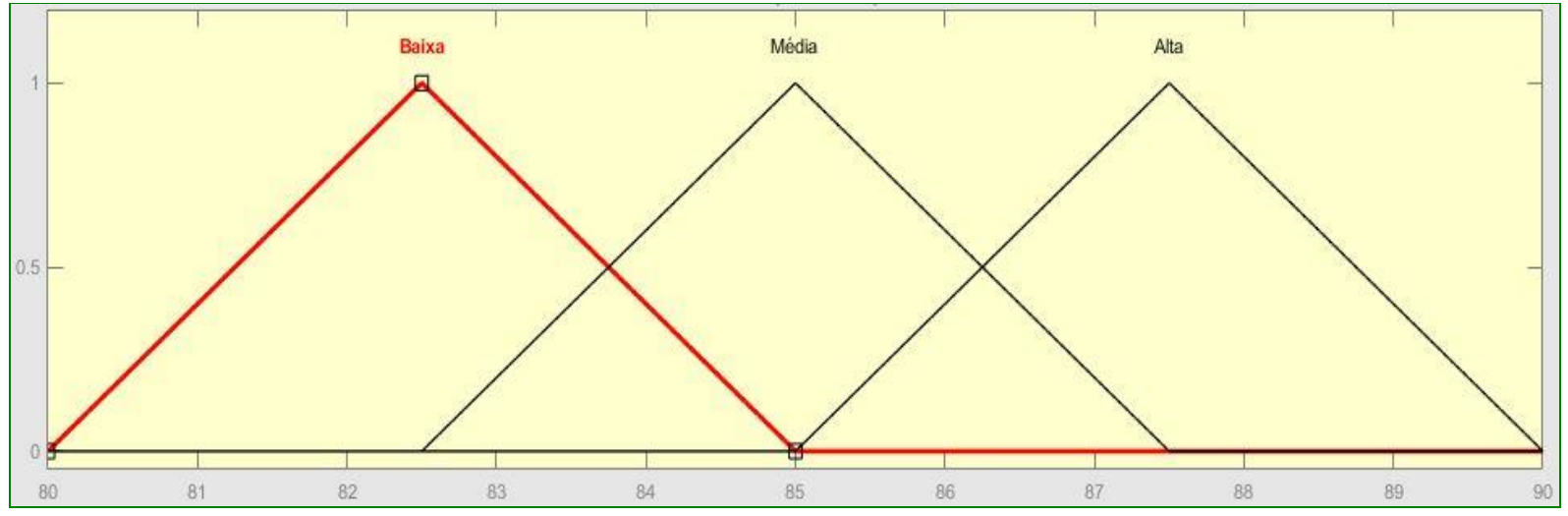

Fonte: Autor, 2021.

A variável de saída foi chamada de Lignina, gerando assim um número real o intervalo $[9,5$ 11,5]. Os graus de pertinência da variável foram chamados de alta deslignificação $(A)$, média deslignificação $(M)$ e baixa deslignificação $(B)$, conforme a Tabela 2 e a Figura 4.

Tabela 2 - Definição do grau de pertinência para a variável Lignina.

\begin{tabular}{c|c|c}
\hline Conjunto difuso & Tipo & Temperatura \\
\hline “Alta deslignificação" A & Triangular & {$[9,510,010,5]$} \\
\hline "Média deslignificação" M & Triangular & {$[10,010,511,0]$} \\
\hline "Baixa deslignificação" B & Triangular & {$[10,511,011,5]$} \\
\hline
\end{tabular}

Fonte: Autor, 2021.

Figura 4 - Grau de associação para conjuntos difusos da variável de saída Lignina.

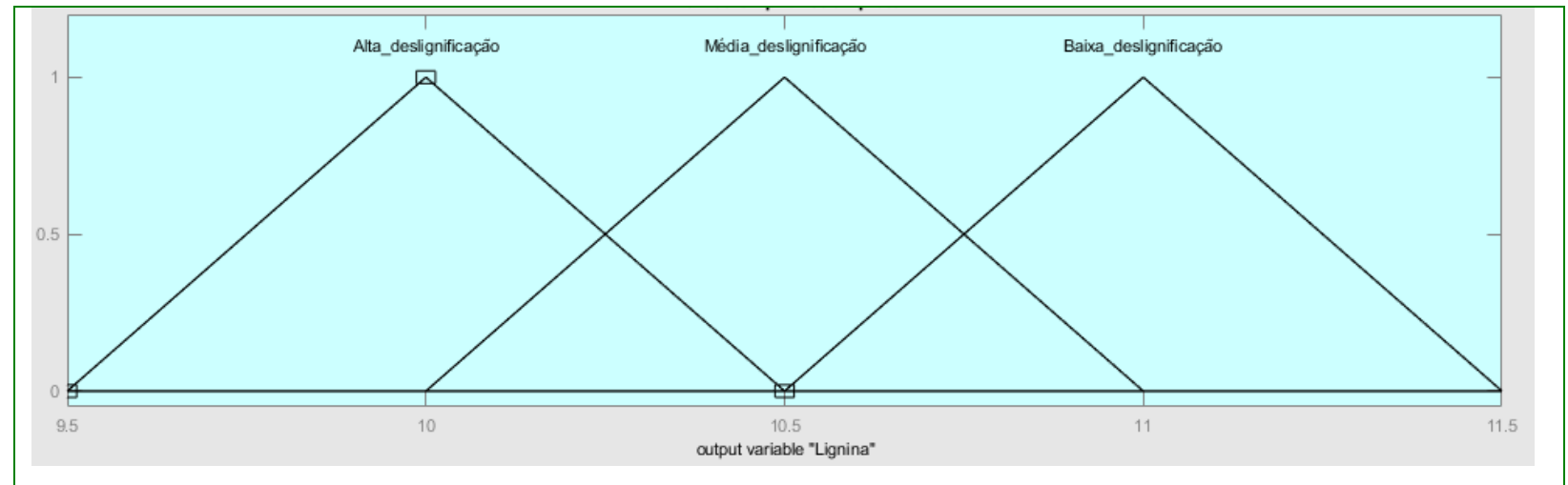

Fonte: Autor, 2021.

Com os dados, foi desenvolvido nove combinações, sendo $(3 \times 3)$ e assim levados ao conjunto fuzzy de duas variáveis de entrada para uma saída. As combinações foram 
realizadas com os graus de pertinência $(B, M$ e $A)$ para a Temperatura e Concentração na entrada e Lignina na saída, conforme a Tabela 3.

Tabela 3 - Combinações das variáveis de entrada e a saída com escalas para graus de adesão 1 associados aos conjuntos fuzzy para a geração da base de regras.

\begin{tabular}{c|c|c}
\hline Concentração (\%) & Temperatura $\left({ }^{\circ} \mathbf{C}\right)$ & Lignina (\%) \\
\hline $\mathrm{B}$ & $\mathrm{B}$ & $\mathrm{M}$ \\
$\mathrm{B}$ & $\mathrm{M}$ & $\mathrm{A}$ \\
\hline $\mathrm{B}$ & $\mathrm{A}$ & $\mathrm{A}$ \\
\hline $\mathrm{M}$ & $\mathrm{B}$ & $\mathrm{B}$ \\
\hline $\mathrm{M}$ & $\mathrm{M}$ & $\mathrm{A}$ \\
\hline $\mathrm{M}$ & $\mathrm{A}$ & $\mathrm{M}$ \\
\hline $\mathrm{A}$ & $\mathrm{B}$ & $\mathrm{B}$ \\
\hline $\mathrm{A}$ & $\mathrm{M}$ & $\mathrm{M}$ \\
\hline
\end{tabular}

Para variáveis de entrada: concentração e temperatura - B: Baixa; M: Média; A: Alta. Para a variável de saída: lignina - A: Alta deslignificação; M: Média deslignificação; B: Baixa deslignificação.

Fonte: Autor, 2021.

O sistema foi baseado em regras difusas estabelecido pela Fuzzy Logic Toolbox de MATLAB® 7.0 (MathWorks Inc. Copyright 1984-2004), acoplado à superfície e do mapa de contorno.

\section{RESULTADOS E DISCUSSÃO}

A Figura 5 apresenta a superfície tridimensional como resposta ao sistema fuzzy desenvolvido utilizando a inferência de Mamdani. Método, com mapa de contorno ilustrado na Figura 6.

Figura 5 - Deslignificação do capim-elefante pré-tratada com NaOH em 3D.

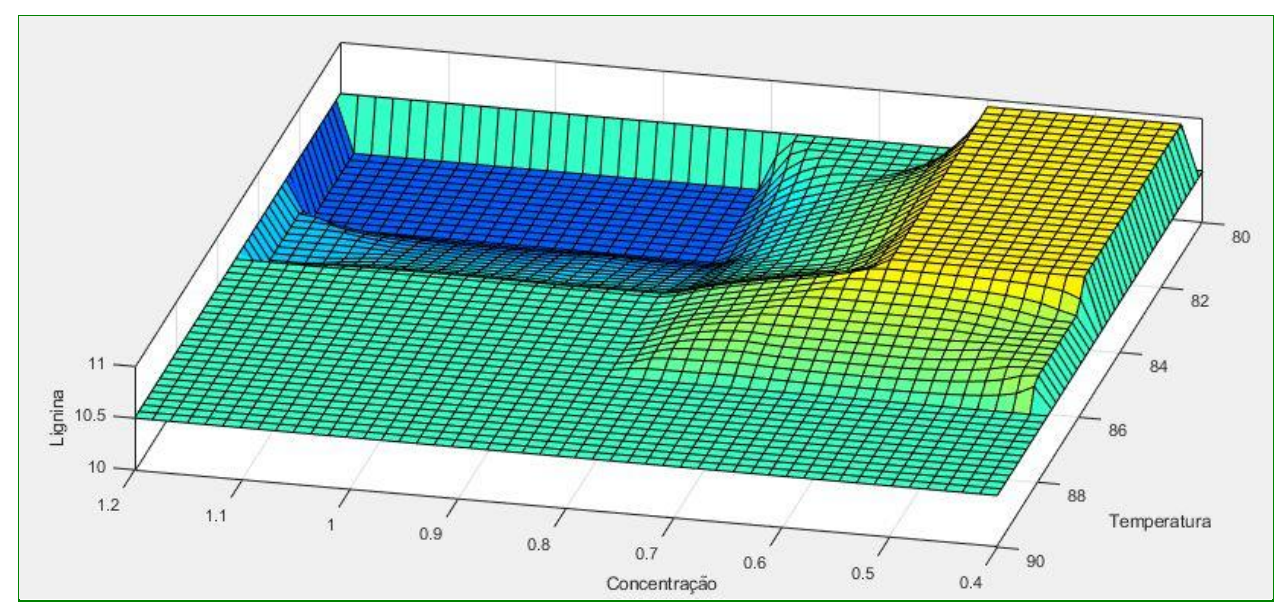

Fonte: Autor, 2021. 
Figura 6 - Mapa de contorno da Lignina na superfície difusa.

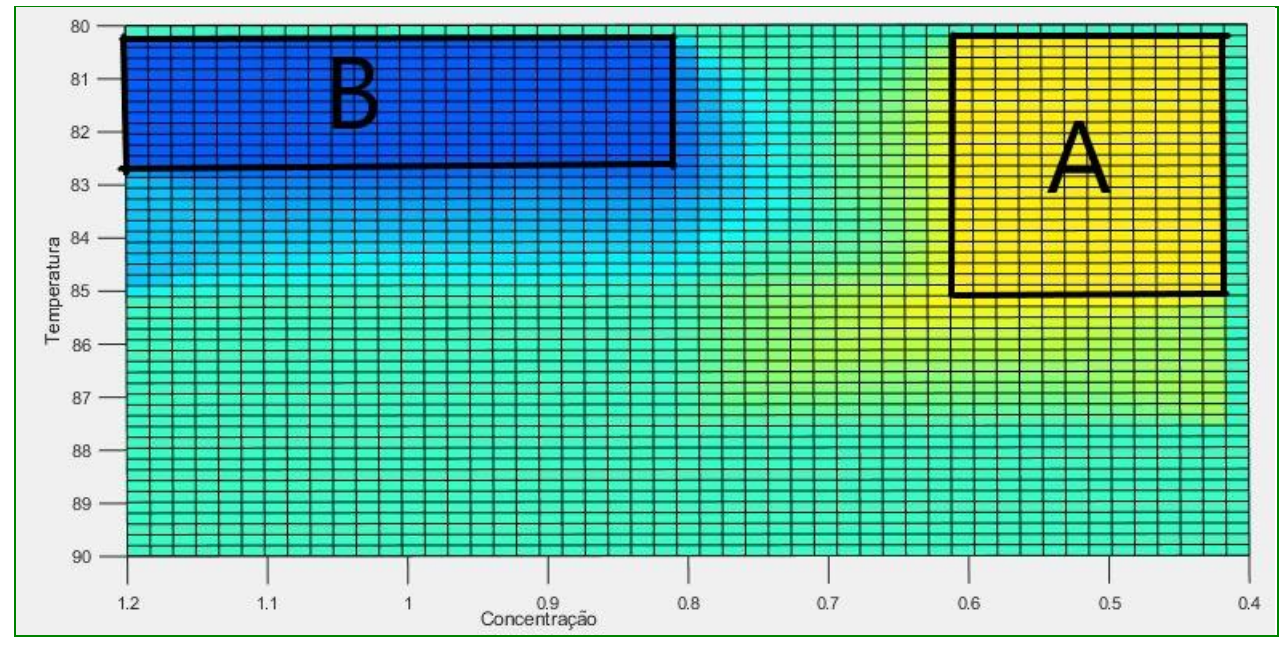

Fonte: Autor, 2021.

A Figura 6 apresenta as duas regiões no plano da Temperatura $\times$ Concentração, e indica onde estão apresentados os melhores pontos para obter melhores resultados na aplicação do pré-tratamento com $\mathrm{NaOH}$ no capim-elefante.

A região A representa as melhores condições as dosagens da concentração de $\mathrm{NaOH}$ e temperatura para o pré-tratamento alcalino de $\mathrm{NaOH}$ no capim-elefante, ou seja, para a temperatura o intervalo desejável está entre 80,5 a $85,0{ }^{\circ} \mathrm{C}$ e para a concentração de $\mathrm{NaOH} 0,4$ a $0,6 \%$.

A região $B$ apresenta dados menos favoráveis para o processo de deslignificação, mesmo possuindo cores mais fortes, que não significa melhores pontos. Estes pontos para temperatura entre 80,5 a $83,5^{\circ} \mathrm{C}$ e para a concentração de $\mathrm{NaOH}$ entre 0,8 a $1,2 \%$.

Pensri et al. (2016) apresentaram resultados que corroboram com os dados da Figura 6 , onde os autores trabalharam com a mesma biomassa utilizando 3 diferentes concentrações de $\mathrm{NaOH}$ no pré-tratamento. De acordo com a literatura citada, os teores de lignina ficaram entre 13,9 a 53,1\%, conforme Tabela 4.

Tabela 4 - Caracterização do capim-elefante pré-tratada com $\mathrm{NaOH}$.

\begin{tabular}{c|c|c}
\hline Amostra & Lignina (\%) & Referência \\
\hline Pré-tratada com NaOH a 1\% & 53,1 & Pensri et al. (2016) \\
\hline Pré-tratada com NaOH a 2\% & 28,1 & Pensri et al. (2016) \\
\hline Pré-tratada com NaOH a 3\% & 13,9 & Pensri et al. (2016) \\
\hline
\end{tabular}

Fonte: Adaptado de Pensri et al., 2016. 
Comparando os resultados de lignina citados na Tabela 4, observa-se que para uma mesma biomassa utilizada (capim-elefante), obtidos neste trabalho com a modelagem no pré-tratada com $\mathrm{NaOH}$ se mostrou superior ao apresentado pelo autor.

Gouveia et al. (2009) utilizaram o bagaço de cana-de-açúcar como biomassa em um processo de pré-tratamento com $\mathrm{NaOH}$ na mesma concentração e 3 momentos diferentes, sendo $1 \%$ de $\mathrm{NaOH}$ a $15 \mathrm{~min} ; 1 \% \mathrm{NaOH}$ a 30 min e $1 \% \mathrm{NaOH}$ a 60 min, onde ocorreu a maior deslignificação com perdas de até $80 \%$, comparando com os resultados da Figura 6 , reafirmou que esta pesquisa mostrou melhores resultados.

A Figura 7 simula o sistema com base em regras difusas para taxas de Temperatura e Concentração. Existindo, uma possibilidade de transição para melhor ponto próximo ao ponto $\mathrm{A}$, com valores entre 0,4 a $0,8 \%$ e 80,5 a $87,0^{\circ} \mathrm{C}$ para concentração e temperatura, respectivamente. O principal ponto dentro da condição excelente é de $0,8 \%$ e $85^{\circ} \mathrm{C}$, conforme Figura 7.

Associando as variáveis de entrada com a saída, foi reconhecido que o ponto de maior grau de pertinência dentro deste conjunto difuso foi para uma concentração Baixa (B) e para temperatura Média (M), resultando em Média (M) para a lignina, como mostra a Figura 8.

Figura 7 - Método de inferência de Mandani para Concentração $=0,8$ e Temperatura $=85,0^{\circ} \mathrm{C}$, com taxa de Lignina difusa $=10,50$.

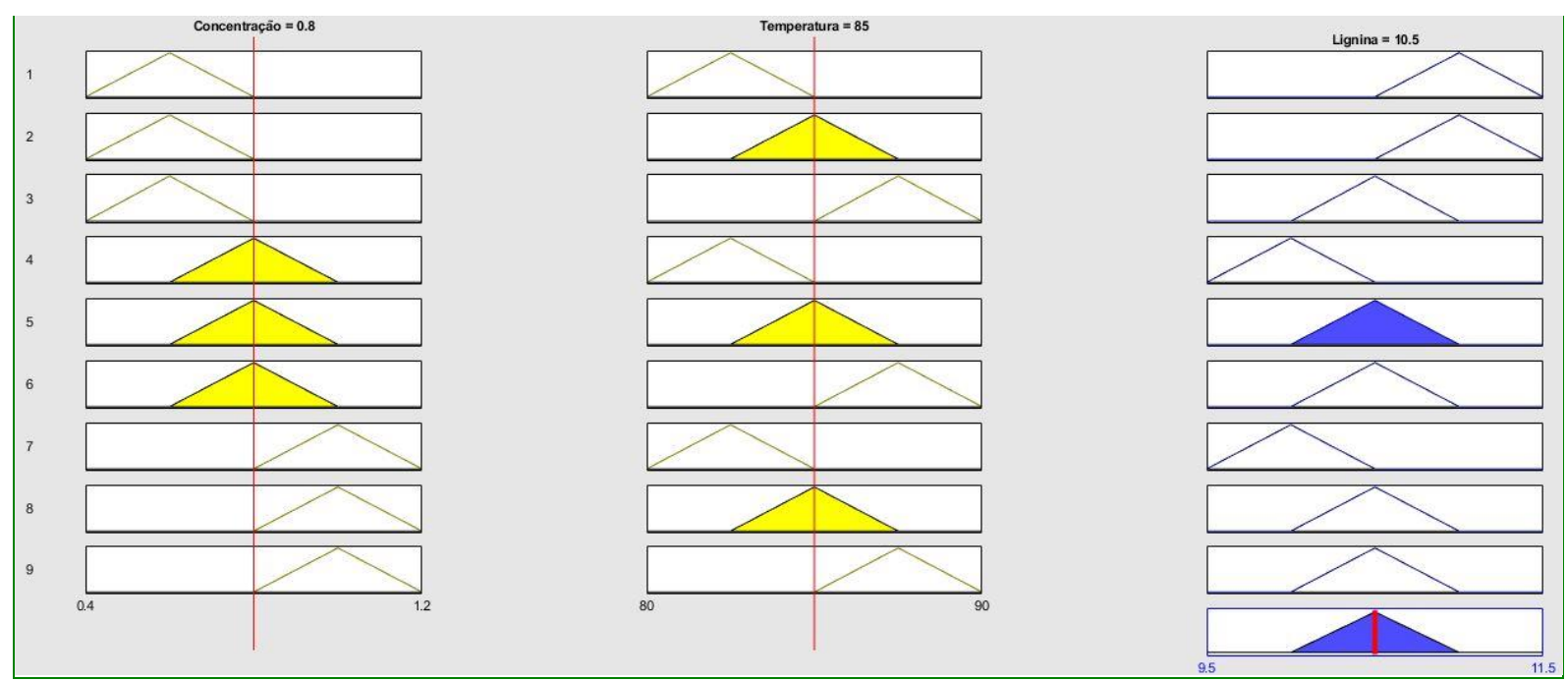

Fonte: Autor, 2021. 
Figura 8 - Indicação de maior grau de pertinência para o conjunto difuso no ponto Médio para Lignina = 10,50 .

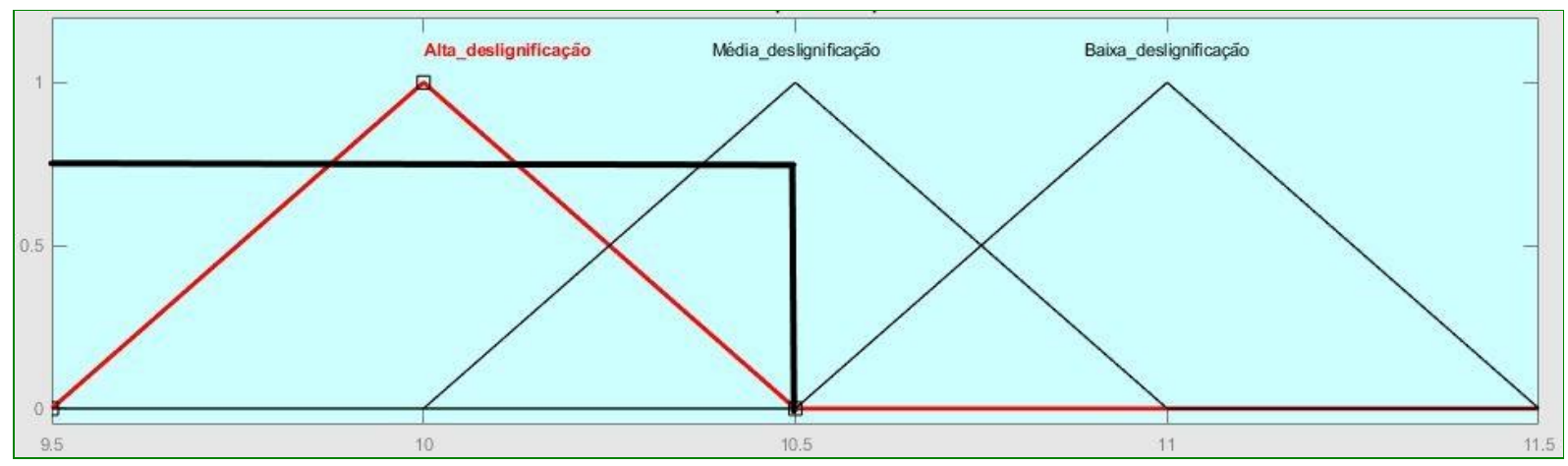

Fonte: Autor, 2021.

Na Figura 8, observa-se que o ponto de deslignificação foi igual a 10,5\%, com a concentração de 0,8\% e temperatura $85 \stackrel{\circ}{\circ}$, Rabemanolontsoa \& Saka (2016) constataram valores de temperaturas acima de $88^{\circ} \mathrm{C}$ e, de concentração acima de $0,8 \%$ pode não ter boas respostas no desempenho desta variável. De maneira similar, Yasuda et al. (2014), constataram baixa deslignificação com a utilização de concentração de $\mathrm{NaOH}$ acima de $1,5 \%$.

\section{CONCLUSÕES}

A lógica fuzzy é eficiente para a busca de dados mais precisos em diversos processos internos, o que possibilita maiores intervalos pré-definidos comercialmente. Com isso, apresenta-se eficiente o processo de pré-tratamento em biomassa principalmente para o capim-elefante no processo de deslignificação. Ainda assim, é uma metodologia que ainda deve ser estudada e que permite reprodução simultânea por muitos pesquisadores.

Levando em consideração que uma biomassa ideal para a produção de biocombustível é aquela que apresenta menor teor de lignina, observou-se que o melhor resultado em deslignificação foi $10,5 \%$ com a concentração $0,8 \%$ e com o uso da temperatura a $85^{\circ} \mathrm{C}$.

\section{REFERÊNCIAS}

BEHERA, S.; ARORA, R.; NANDHAGOPAL, N.; KUMAR, S. Importance of chemical pretreatment for bioconversion of lignocellulosic biomass. Renewable and Sustainable Energy Reviews, v. 36, p. 91-106, 2014. DOI: https://doi.org/10.1016/j.rser.2014.04.047. 
BHUTTO, A.; HARIJAN, K.; QURESHI, K.; BAZMI, A. A.; BAHADORI, A. Perspectives for the production of ethanol from lignocellulosic feedstock - A case study. Journal of Cleaner Production, v. 95, p. 184-193, 2015. DOI:

https://doi.org/10.1016/j.jclepro.2015.02.091.

CANEPPELE, F. de L.; GODINHO, E. Z.; ZUIN, L. F. L.; GABRIEL FILHO, L. R. A. Aplicação da lógica fuzzy no desenvolvimento do morango no Oeste do Paraná. Revista Sodebras, v. 16, n. 184, p. 6-9, 2021. DOI: https://doi.org/10.29367/issn.18093957.16.2021.184.06.

CARDONA, E.; RIOS, J.; PEÑA, R.; RIOS, J. Effects of the pretreatment method on enzymatic hydrolysis and ethanol fermentability of the cellulosic fraction from elephant grass. Fuel, v. 118, p. 41-47, 2014. DOI: https://doi.org/10.1016/j.fuel.2013.10.055.

CARDONA, E.; RIOS, J.; PEÑA, R.; RIOS, J. Pretratamiento Alcalino de Pasto Elefante (Pennisetum sp) y King Grass (Pennisetum hybridum) Cultivados en Colombia para la Producción de Bioetanol. Informacion Tecnologica, v. 24, n. 5, p. 69-80, 2013. DOI: https://dx.doi.org/10.4067/S0718-07642013000500009.

CARVALHO-NETTO, O. V.; BRESSIANI, J. A.; SORIANO, H. L. et al. The potential of the energy cane as the main biomass crop for the cellulosic industry. Chemical and Biological Technologies in Agriculture, p. 1-20, 2014. DOI:

https://doi.org/10.1186/s40538-014-0020-2.

DEL RÍO, J. C.; LINO, A. G.; COLODETTE, J. L.; LIMA, C. F.; GUTIÉRREZ, A.; MARTÍNEZ, Á. T.; LU, F.; RALPH, J.; RENCORET, J. Differences in the chemical structure of the lignins from sugarcane bagasse and straw. Biomass and Bioenergy, v. 81, p. 322-338, 2015. DOI: https://doi.org/10.1016/j.biombioe.2015.07.006.

GODINHO, E. Z.; PERIN, A.; BAUMGARTNER, T. R. da S.; HASAN, S. D. M. Prétratamento hidrotérmico alcalino e alcalino-oxidativo sobre os teores de celulose e lignina em biomassa de capim elefante BRS Capiaçu. Journal of Bioenergy and Food Science, v. 6, n. 3, p. 51-65, 2019. DOI: https://doi.org/10.18067/jbfs.v6i3.263.

GODINHO, E. Z.; CANEPPELE, F. de L.; GASPAROTTO, H. V. Utilização da lógica fuzzy para otimizar aplicação de fertilizantes no rabanete. Brazilian Journal of Biosystems Engineering, v. 15, n. 2, p. 270-282, 2021. DOI:

http://dx.doi.org/10.18011/bioeng2021v15n2p270-282.

GODOY, F. O. de.; GODINHO, E. Z.; DALTIN, R. S.; CANEPPELE, F. de L. Utilização da lógica fuzzy aplicada à energia solar. Cadernos de Ciência \& Tecnologia, v. 37, n. 2, p. 26663, 2020. DOI: http://dx.doi.org/10.35977/0104-1096.cct2020.v37.26663.

GOUVEIA, E. R. et al. Validação de metodologia para a caracterização química de bagaço de cana-de-açúcar. Química Nova, v. 32, n. 6, p. 1500-1503, 2009.

https://doi.org/10.1590/S0100-40422009000600026. 
MANEKAR, P. NANDY, T.; SARGAONKAR, A.; RATHI, B.; KARTHIK, M. FAHP ranking and selection of pretreatment module for membrane separation processes in textile cluster. Bioresource Technology, v. 102, n. 2, p. 558-566, 2011.

https://doi.org/10.1016/i.biortech.2010.07.086.

PENSRI, B.; AGGARANGSI, P.; CHAIYASO, T.; CHANDET, N. Potential of Fermentable Sugar Production from Napier cv. Pakchong 1 Grass Residue as a Substrate to Produce Bioethanol. Energy Procedia, v. 89, p. 428-436, 2016. DOI:

https://doi.org/10.1016/j.egypro.2016.06.287.

PUSPAWATI, S.; WAGIMAN, M. A.; DARMAWAN, A. N.; HASLIANTI, A. The Production of Bioethanol Fermentation Substrate from Eucheuma cottonii Seaweed through Hydrolysis by Cellulose Enzyme. Agriculture and Agricultural Science Procedia, v. 3, p. 200-205, 2015. DOI: https://doi.org/10.1016/i.aaspro.2015.01.039.

RABEMANOLONTSOA, H.; SAKA, S. Various pretreatments of lignocellulosics. Bioresource Technology, v. 199, p. 83-91, 2016.

RUEDA, J. A.; ORTEGA-JIMÉNEZ, E.; HERNÁNDEZ-GARAY, A.; ENRÍQUEZ-QUIROZ, J. F.; GUERRERO-RODRÍGUEZ, J. D.; QUERO-CARRILLO, A. R. Growth, yield, fiber content and lodging resistance in eight varieties of Cenchrus purpureus (Schumach.) Morrone intended as energy crop. Biomass and Bioenergy, v. 88, p. 59-65, 2016. DOI: https://doi.org/10.1016/j.biombioe.2016.03.007.

YASUDA, M.; NAGAI, H.; TAKEO, K.; ISHII, Y.; OHTA, K. Bio-ethanol production through simultaneous saccharification and co-fermentation (SSCF) of a low-moisture anhydrous ammonia (LMAA)-pretreated napiegrass (Pennisetum purpureum Schumach). SpringerPlus, v. 3, n. 1, 2014. DOI: https://doi.org/10.1186/2193-1801-3-333. 
ABSTRACT

Elephant grass has great potential for the production of bioethanol, with emphasis on field production and industrial handling. The pre-treatment process in biomass is essential to obtain high yields in bioethanol production. Therefore, optimization is essential for cost reductions and increased financial profitability. As a result, it aimed to develop a system based on fuzzy rules, which optimized the pre-treatment process with $\mathrm{NaOH}$ in elephant grass. The fuzzy model was developed using two input and one output variables, namely concentration, temperature and lignin, respectively. Analysis of the effects of concentration and temperature was performed using three-dimensional surfaces and contour maps of the output variable. It was found that the variables related to lignin are largely affected by a low dose of $\mathrm{NaOH}$ concentration and with a medium to low temperature. In general, it is concluded that the region between 0.4 to $0.6 \%$ of $\mathrm{NaOH}$ concentration, combined with a temperature between 80.5 to $85.0^{\circ} \mathrm{C}$, the best results are verified for the studied biometric variable, lignin.

Keywords: Lignin. Sodium hydroxide. Fuzzy Logic.

\section{RESUMEN}

El proceso de pretratamiento en biomasa es fundamental para obtener altos rendimientos en la producción de bioetanol. Por lo tanto, la optimización es esencial para reducir costos y aumentar la rentabilidad financiera. Como resultado, se propuso desarrollar un sistema basado en reglas difusas, que optimizara el proceso de pretratamiento con $\mathrm{NaOH}$ en pasto elefante. El modelo difuso se desarrolló utilizando dos variables de entrada y una de salida, a saber, concentración, temperatura y lignina, respectivamente. El análisis de los efectos de la concentración y la temperatura se realizó utilizando superficies tridimensionales y mapas de contorno de la variable de salida. Se encontró que las variables relacionadas con la lignina se ven afectadas en gran medida por una dosis baja de concentración de $\mathrm{NaOH}$ y con una temperatura media a baja. En general, se concluye que la región entre 0.4 a $0.6 \%$ de concentración de $\mathrm{NaOH}$, combinada con una temperatura entre 80.5 a $85.0^{\circ} \mathrm{C}$, se verifican las mayores y mejores respuestas de las variables biométricas estudiadas.

Palabras-clave: Lignina. Hidróxido de sódio. Lógica difusa. 


\section{LICENÇA DE USO}

Este é um artigo publicado em acesso aberto (Open Access) sob a licença Creative Commons Atribuição 4.0 Internacional (CC BY 4.0), que permite uso, distribuição e reprodução em qualquer meio, desde que o trabalho original seja corretamente citado. Mais informações em: http://creativecommons.org/licenses/by/4.0

\section{CONFLITO DE INTERESSES}

Os autores declaram que não há conflito de interesses neste trabalho.

\section{CONTRIBUIÇÕES AUTORAIS}

Autor 1: Responsável (montagem, execução dos trabalhos e dados estatísticos) do experimento (referência) desde o plantio do capim-elefante até o processo de deslignificação da biomassa, também responsável pela montagem e coleta dos dados para o artigo no modelo da revista, aplicou os dados no software, fez os resultados e discussão e a conclusão.

Autor 2: Supervisão geral do artigo, correções gerais.

Autor 3: Organização do artigo na questão da introdução, tradução do resumo para o inglês e revisão do artigo.

\section{FINANCIAMENTO}

O presente trabalho não contou com apoio financeiro.

\section{COMO REFERENCIAR}

GODINHO, Emmanuel Zullo; CANEPPELE, Fernando de Lima; GASPAROTTO, Hélio Vagner. Aplicabilidade da lógica nebulosa no processo de deslignificação do capimelefante no pré-tratamento com NAOH. Revista Brasileira de Engenharia de Biossistemas (Tupã), v. 15, n. 3, p. 497-510, 2021. DOI: http://dx.doi.org/10.18011/bioeng2021v15n3p497-510.

\section{RESPONSABILIBADE EDITORIAL}

Prof. Dr. Fernando Ferrari Putti ${ }^{1}$, Prof. Dr. Paulo Sérgio Barbosa dos Santos ${ }^{1}$, Prof. Dr. Eduardo Festozo Vicente ${ }^{1}$ e Prof. Dr. Diogo de Lucca Sartori ${ }^{1}$

${ }^{1}$ Universidade Estadual Paulista "Júlio de Mesquita Filho", FCE - Faculdade de Ciências e Engenharia, Tupã, SP, Brasil. 